\title{
Autosomal dominant mannose-binding lectin deficiency is associated with worse neurodevelopmental outcomes after cardiac surgery in infants
}

\author{
Daniel Seung Kim, PhD, MPH, ${ }^{\mathrm{a}, \mathrm{b}, \mathrm{c}}$ Yatong K. Li, AB, ${ }^{\mathrm{c}}$ Jerry H. Kim, MD, MPH, ${ }^{\mathrm{d}}$ Curtis S. Bergquist, MD, \\ Marsha Gerdes, PhD,${ }^{f}$ Judy C. Bernbaum, MD, ${ }^{f}$ Nancy Burnham, MSN,${ }^{g}$ \\ Donna M. McDonald-McGinn, MS, ${ }^{\mathrm{h}}$ Elaine H. Zackai, MD, ${ }^{\mathrm{h}}$ Susan C. Nicolson, MD, \\ Thomas L. Spray, MD, ${ }^{\mathrm{g}}$ Deborah A. Nickerson, PhD, ${ }^{\mathrm{b}}$ Hakon Hakonarson, MD, PhD, ${ }^{\mathrm{j}}$ \\ Gail P. Jarvik, MD, PhD, ${ }^{\mathrm{a}, \mathrm{b}}$ and J. William Gaynor, $\mathrm{MD}^{\mathrm{g}}$
}

\section{ABSTRACT}

Objectives: The $M B L 2$ gene is the major genetic determinant of mannose-binding lectin (MBL) - an acute phase reactant. Low MBL levels have been associated with adverse outcomes in preterm infants. The $M B L 2_{\text {Gly54Asp }}$ missense variant causes autosomal dominant MBL deficiency. We tested the hypothesis that $M B L 2_{G l y 54 A s p}$ is associated with worse neurodevelopmental outcomes after cardiac surgery in neonates.

Methods: This is an analysis of a previously described cohort of patients with nonsyndromic congenital heart disease who underwent cardiac surgery with cardiopulmonary bypass before age 6 months $(n=295)$. Four-year neurodevelopment was assessed in 3 domains: Full-Scale Intellectual Quotient, the Visual Motor Integration development test, and the Child Behavior Checklist to assess behavior problems. The Child Behavior Checklist measured total behavior problems, pervasive developmental problems, and internalizing/externalizing problems. A multivariable linear regression model, adjusting for confounders, was fit.

Results: $M B L 2_{G l y 54 A s p}$ was associated with a significantly increased covariateadjusted pervasive developmental problem score $(\beta=3.98 ; P=.0025)$. Sensitivity analyses of the interaction between age at first surgery and MBL genotype suggested effect modification for the patients with $M B L 2_{\text {Gly54Asp }}$ $\left(P_{\text {interaction }}=.039\right)$, with the poorest neurodevelopment outcomes occurring in children who had surgery earlier in life.

Conclusions: We report the novel finding that carriers of $M B L 2_{\text {Gly54Asp }}$ causing autosomal dominant MBL deficiency have increased childhood pervasive developmental problems after cardiac surgery, independent of other covariates. Sensitivity analyses suggest that this effect may be larger in children who underwent surgery at earlier ages. These data support the role of nonsyndromic genetic variation in determining postsurgical neurodevelopment-related outcomes in children with congenital heart disease. (J Thorac Cardiovasc Surg 2018;155:1139-47)

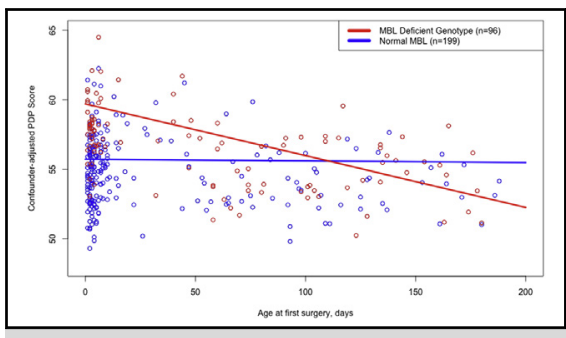

Mannose-binding lectin (MBL) deficiency and early surgery combined increase pervasive developmental problems.

\section{Central Message}

Autosomal dominant MBL deficiency is associated with worse neurodevelopmental outcomes after surgery for congenital heart disease. Neuroprotection is important as survival in patients increases.

\section{Perspective}

As survival for congenital heart surgery improves, the need for decreased morbidity has increased. We identify in this work a genetic variant that causes autosomal dominant mannose binding lectin deficiency and report its association with worse neurodevelopment. Its effects were exacerbated in younger patients. These findings could help in risk stratification to reduce morbidity.

See Editorial Commentary page 1148.

See Editorial page 1138.
From the ${ }^{\mathrm{a}}$ Division of Medical Genetics, Department of Medicine, and Departments
of ${ }^{\mathrm{b}}$ Genome Sciences and ${ }^{\mathrm{d}}$ Anesthesiology and Pain Medicine, University of Wash-
ington, Seattle, Wash; the ${ }^{\mathrm{c}}$ Department of Biostatistics, and ${ }^{\mathrm{e}}$ Section of Thoracic
Surgery, Department of Surgery, University of Michigan, Ann Arbor, Mich; and
the ${ }^{\mathrm{f}}$ Department of Pediatrics, and Divisions of ${ }^{\mathrm{g}}$ Cardiothoracic Surgery, ${ }^{\mathrm{h}}$ Genetics,
and ${ }^{\mathrm{i}}$ Cardiothoracic Anesthesiology, and the ${ }^{\mathrm{j}}$ Center for Applied Genomics, The
Children's Hospital of Philadelphia and the Perelman School of Medicine, Univer-
sity of Pennsylvania, Philadelphia, Pa.
Supported by a grant from the Fannie E. Rippel Foundation, an American Heart As-
sociation National Grant-in-Aid (No. $9950480 \mathrm{~N})$, National Institutes of Health
grant No. HL071834, and a Washington State Life Sciences Discovery Award to
the Northwest Institute for Genetic Medicine. Dr D. Kim was supported by Na-
tional Institutes of Health grants Nos. 1F31MH101905-01 and T32HL007312 and American Hospital Association grant No. 16POST27250048. Dr J. Kim was supported by National Center for Research Resources grant No. KL2 TR000421. Read at the 97th Annual Meeting of The American Association for Thoracic Surgery, Boston, Massachusetts, April 29-May 3, 2017.

Received for publication Feb 24, 2017; revisions received July 1, 2017; accepted for publication Aug 3, 2017.

Address for reprints: J. William Gaynor, MD, Division of Cardiothoracic Surgery, Rm 8527 Main Building, 34th St and Civic Center Blvd, Philadelphia, PA 19104-4399 (E-mail: gaynor@email.chop.edu).

$0022-5223 / \$ 36.00$

Copyright (C) 2017 by The American Association for Thoracic Surgery https://doi.org/10.1016/j.jtcvs.2017.08.035 


\section{Abbreviations and Acronyms \\ APOE = apolipoprotein $\mathrm{E}$ \\ CBCL $1.5-5=$ Child Behavior Checklist for ages \\ 1.5 to 5 years \\ CHD $=$ congenital heart disease \\ CHOP $=$ Children's Hospital of Philadelphia \\ $\mathrm{CPB}=$ cardiopulmonary bypass \\ DHCA $=$ deep hypothermic circulatory arrest \\ FSIQ = full-scale intelligence quotient \\ LOS = length of stay \\ MBL $=$ mannose-binding lectin \\ PDP = pervasive developmental problems \\ VMI $=$ visual-motor integration}

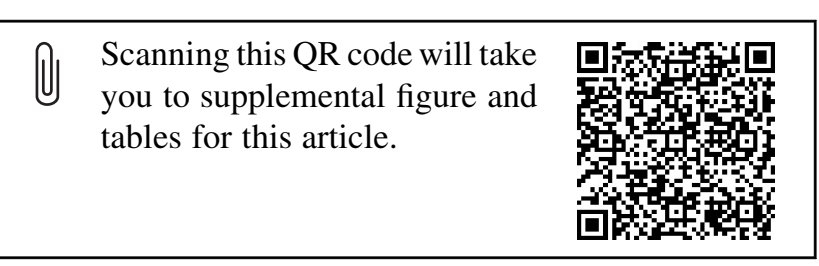

Congenital heart disease (CHD) is the most common human birth defect, frequently requiring surgical intervention with cardiopulmonary bypass (CPB) or circulatory arrest soon after birth. Although survival has improved, studies have identified a higher incidence of adverse neurologic and functional outcomes in survivors of neonatal surgery for CHD. ${ }^{1,2}$ Neurodevelopmental dysfunction is the most common adverse postsurgical outcome, with approximately $15 \%$ and $30 \%$ of survivors showing symptoms of pervasive developmental problems (PDPs) and attention-deficit hyperactivity disorder, respectively, by age 5 years. ${ }^{3}$

Mannose-binding lectin (MBL) is a hepatic-derived acute phase reactant that activates the complement system. ${ }^{4}$ Serum levels of MBL in humans are entirely determined by the $M B L 2$ gene (MBL1 is a nonfunctional pseudogene). ${ }^{5}$ Prior work identified a glycine-to-aspartate missense variation at the 54th codon of the MBL2 protein $\left(M B L 2_{\text {Gly } 54 A s p}\right.$, rs1800450, population minor allele frequency approximately $14 \%$ ) as the cause of autosomal dominant MBL deficiency (Mendelian Inheritance in Man No. 614372). These patients manifest recurrent neonatal infections. ${ }^{6,7} \mathrm{~A}$ single copy of $M B L 2_{\text {Gly54Asp }}$ decreases serum MBL activity by approximately $90 \%$ - to the level of those with 2 copies of $M B L 2_{\text {Gly54Asp }},{ }^{6,7}$ likely through a dominant-negative effect, whereby proteins with the aspartate protein change interfere with oligomerization of MBL into the mature, polymerized serum molecule that is then involved in carbohydrate recognition and initiation of the complement cascade. $^{8}$ In addition, a single copy of $M B L 2_{\text {Gly54Asp }}$ has been associated with increased prevalence of sepsis in neonates ${ }^{9}$ and adverse neurologic outcomes in preterm infants. ${ }^{10}$

Given the importance of MBL in immunity for neonates and its potential link to adverse neurologic events, we hypothesize that $M B L 2_{\text {Gly } 54 A s p}$ also affects neurodevelopment. Thus, in this study we sought to determine whether autosomal dominant MBL deficiency, as caused by $M B L 2_{G l y 54 A s p}$, is associated with differential neurodevelopmental outcomes at 4-year follow-up in children with isolated CHD who required surgical palliation.

\section{METHODS \\ Ethics Statement}

Participants were enrolled at the Children's Hospital of Philadelphia (CHOP) on a protocol approved by the institutional review boards of CHOP and the University of Washington (analysis) from October 1998 to April 2003. Informed, written consent was obtained from parents or guardians of all participants.

\section{Patient Population}

This is an analysis of a previously described prospective cohort of 550 participants enrolled in a prospective study at the CHOP to study neurodevelopmental dysfunction after surgical correction for CHD. ${ }^{1-14}$ Patients aged 6 months or younger who underwent CPB with or without deep hypothermic circulatory arrest (DHCA) for repair of CHD were eligible for enrollment. Exclusion criteria included multiple distinct congenital anomalies, a likely genetic or phenotypic syndrome, and a language other than English spoken in the home. This study examined a subset of the cohort with both genetic and 4-year neurodevelopmental outcome data $(\mathrm{n}=295)$.

Of the original $550 \mathrm{CHD}$ cases, 381 returned for 4-year neurodevelopment assessment. An additional 53 participants were excluded due to presence of chromosomal/genetic abnormalities (eg, DiGeorge/22q11.2 deletion syndrome) that would be expected to bias the results due to high prevalence of poor neurodevelopmental outcomes compared with nonsyndromic CHD patients. ${ }^{3,15}$ Thirty-three patients were excluded due to a lack of high quality genotype data. There were 295 patients considered after these exclusions. Information on data collection (including further information on inclusion/exclusion criteria) operative management, and genotyping have been previously reported in detail. ${ }^{12}$

\section{Genetic Evaluation to Exclude Syndromic CHD Participants}

CHD participants were evaluated by a genetic dysmorphologist and genetic counselor at the 1-year and/or 4-year examinations. Patients were classified as having no indication of genetic syndrome or chromosomal abnormality (normal, isolated CHD), suspected genetic syndrome (suspect), or a definite genetic syndrome or chromosomal abnormality (genetic). Following this classification, genetics records for each patient with a CHD were individually reviewed by a second senior board-certified medical geneticist, blinded to the genetic data, to determine whether participants were to be included or excluded from the current analysis, which focuses on nonsyndromic participants. Due to this review, 53 CHD participants with known or suspected genetic syndromes were excluded from analysis due to the potential for genetic confounding effects on neurodevelopmental outcomes. ${ }^{3,15}$ 


\section{Four-Year Neurodevelopmental Examinations}

Neurodevelopmental evaluations were performed between the fourth and fifth birthdays. Growth measurements (ie, weight, length, and head circumference) were recorded and a health history was obtained, focusing on the incidence of interim illnesses, hospitalizations, neurologic events or interim evaluations, current medication use, and parental concerns about health. Parents were asked specifically whether they had ever been told that their child had autism, Asperger syndrome, pervasive developmental disorder not otherwise specified, or attention-deficit hyperactivity disorder.

General child intelligence and visual-motor input integration were tested using the Wechsler Preschool and Primary Scale of IntelligenceThird Edition ${ }^{16}$ and the Beery-Buktenica Developmental Test of VisualMotor Integration-Sixth Edition, ${ }^{17}$ respectively. Both the full-scale intelligence quotient (FSIQ) and visual-motor integration (VMI) score are standardized to a mean of $100 \pm 15$.

Behavior skills were assessed through parental report by using the Child Behavior Checklist for ages 1.5 to 5 years (CBCL 1.5-5). ${ }^{18}$ The CBCL 1.55 is a questionnaire used to obtain parental reports of behavior problems demonstrated within the previous 6 months. The PDP score is among the clinically oriented scores resulting from the CBCL $1.5-5$ and has been previously used to identify preschoolers at risk for autism. ${ }^{19}$ Other scores from the CBCL 1.5-5 include a total problem score and 2 broad behavioral indices (ie, internalizing and externalizing problems). The CBCL 1.5-5 total problems score consists of the sum of the scores for the 99 specific problem items on the form plus the highest scores for any written-in responses to item 100. The internalizing problems scores summarize specific scores regarding the behaviors of excessive emotional reactivity, anxious/ depressed behavior, somatic complaints, and withdrawn behavior. The externalizing problems score summarizes the 2 specific behavior scores of attention problems and aggressive behavior. All raw scores (ie, PDP, total problems, externalizing problems, and internalizing problems) were transformed to $T$ scores with mean $=50$ and these $T$ scores were directly used in analyses.

\section{$M B L 2_{G l y 54 A s p}(\mathrm{rs1800450})$ genotyping}

Whole blood or buccal swab samples were obtained before surgery and were stored at $4{ }^{\circ} \mathrm{C}$. Genomic DNA was isolated from white blood cells and genotyping was performed using the Illumina HumanHap550Quad+ BeadChip (Illumina Inc, San Diego, Calif) at the Center for Applied Genomics of CHOP. Patient genotypes at rs1800450, the DNA missense variant responsible for autosomal dominant MBL deficiency, were extracted from the larger, nonimputed genetic dataset using PLINK. ${ }^{20}$

\section{Apolipoprotein E genotyping}

Genomic DNA was prepared and was used for determination of apolipoprotein $\mathrm{E}(\mathrm{APOE})$ genotypes as previously described. ${ }^{11}$ APOE genotypes were classified into 3 groups as follows: $\varepsilon 2$ ( $\varepsilon 2 \varepsilon 2$, $\varepsilon 2 \varepsilon 3$, or $\varepsilon 2 \varepsilon 4) \varepsilon 3$ ( $\varepsilon 3 \varepsilon 3)$, or $\varepsilon 4$ ( $\varepsilon 4 \varepsilon 3$ or $\varepsilon 4 \varepsilon 4)$, and the APOE $\varepsilon 2$ genotype was included as a covariate in the linear regression model for all phenotypes based on considerable prior published evidence that the APOE $\varepsilon 2$ genotype is associated with markedly detrimental neurodevelopmental outcomes. ${ }^{3,11,21}$

\section{Statistical Analyses}

All analyses and graphics were performed in R (R Foundation for Statistical Computing, Vienna, Austria) using standard regression packages.

Genetic ancestry was determined using previously described methods. ${ }^{22}$ Due to the mixed ancestry of the cohort, the first 3 principal component eigenvectors were used as covariates in the linear regression models for neurodevelopmental outcomes to adjust for potential population stratification. $^{23}$

Due to the autosomal dominant inheritance of MBL deficiency, we categorized patients with 1 or more copies of the $M B L 2_{\text {Gly54Asp }}$ missense variant into a single group (hereafter termed MBL-deficient genotype).
The distribution of rs 1800450 genotype among the cohort was 199 homozygous major, 84 heterozygous, and 12 homozygous minor ( 96 total MBLdeficient genotype patients).

Linear regression models were separately fit for the 6 considered neurodevelopmental outcomes (FSIQ, VMI score, and CBCL PDPs, total score, internalizing problems, and externalizing problems) to assess the association of MBL-deficient genotype with each outcome. A Bonferroni-adjusted threshold for significance was set at $\alpha=0.0083$ to adjust for the 6 total neurodevelopmental outcomes tested ( 0.05 out of 6 ). Each linear regression model was adjusted for the previously reported confounding variables: the first 3 principal component eigenvectors for ancestry, gestational age, birth weight, birth head circumference, diagnostic class (coded as a dummy variable with diagnostic class 1 as the reference group), ${ }^{24}$ preoperative intubation, preoperative length of stay (LOS), total minutes of CPB, total minutes of DHCA, delayed sternal closure, extracorporeal membrane oxygenation use, hematocrit at first surgery, postoperative LOS, mother's education at 4-year follow-up, and mother's socioeconomic status at 4year follow-up. Diagnosis class was assigned based on preoperative diagnosis according to a previously proposed scheme ${ }^{24}$ : class I, 2-ventricle heart without arch obstruction; class II, 2-ventricle heart with arch obstruction; class III single-ventricle heart without arch obstruction; and class IV, single-ventricle heart with arch obstruction.

\section{Plotting of Gene $\times$ Environment Interactions}

A secondary analysis considering the possibility of effect modification of MBL deficiency by age at first surgery was fit using linear regression. Visualization of this gene $\times$ environment interaction was obtained through simplification of the linear regression coefficients: [Predicted PDP score $=\alpha+\beta_{1}$ (MBL deficient) $+\beta_{2}$ (age at first surgery) $+\gamma$ (MBL deficient $\times$ age at first surgery)], such that the MBL deficient have coefficients equal to $\alpha+\beta_{1}+\beta_{2}+\gamma$, whereas those with normal MBL status have coefficients equal to $\alpha+\beta_{2}$.

\section{RESULTS}

Baseline characteristics of the studied cohort stratified by MBL status are presented in Table 1. A total of 199 patients had normal MBL status, as determined by their homozygous major genotype at rs 1800450 . An additional 96 participants were classified as having MBL-deficient genotype, as they carried 1 or 2 copies of the minor allele at rs 1800450 (resulting in $M B L 2_{\text {Gly54Asp }}$, the missense variant causative for autosomal dominant MBL deficiency (minor allele frequency $F=13 \%$, see Mendelian Inheritance in Man No. 614372)). When comparing the 2 MBL groups and not adjusting for multiple contrasts, the MBL-deficient group had significant decreases in preoperative LOS (1.62 vs 2.37 days; $P=.002$ ) and postoperative $\operatorname{LOS}(8.85$ vs 11.50 days; $P=.011)$ as well as significant increases in average age at first surgery (57.7 vs 36.3 days; $P=.013$ ). We also report marginal differences in the distribution of maternal education at 4 years of follow-up $(P=.024)$ and in self-reported race $(P=.060)$ between the MBLdeficient genotype and normal MBL groups.

To determine whether MBL status was associated with 4year neurodevelopmental outcomes, a multivariable linear regression model adjusting for numerous potential confounders (see Statistical Analysis) was applied. The differences in the other underlying risk factors between genotype 
TABLE 1. Baseline and clinical characteristics of the 295 participants, stratified by mannose-binding lectin (MBL) status

\begin{tabular}{|c|c|c|c|c|}
\hline & $\mathbf{n}$ & $\begin{array}{l}\text { Normal MBL } \\
\quad(n=199)\end{array}$ & $\begin{array}{l}\text { MBL-deficient genotype } \\
\qquad(\mathbf{n}=96)\end{array}$ & 2 -sided $P$ value \\
\hline Male & 295 & $110(55)$ & $56(58)$ & .62 \\
\hline Gestational age (wk) & 295 & $38.57 \pm 1.87$ & $38.45 \pm 2.40$ & .82 \\
\hline Birth weight (kg) & 295 & $3.17 \pm 0.61$ & $3.14 \pm 0.71$ & .52 \\
\hline Birth head circumference $(\mathrm{cm})$ & 295 & $33.82 \pm 1.49$ & $33.85 \pm 1.71$ & .90 \\
\hline $\begin{array}{l}\text { Race } \\
\text { American Indian/Alaska Native } \\
\text { Asian } \\
\text { Black } \\
\text { Hispanic } \\
\text { White }\end{array}$ & 295 & $\begin{array}{c}4(2) \\
7(4) \\
53(27) \\
8(4) \\
127(64)\end{array}$ & $\begin{array}{c}3(3) \\
4(4) \\
11(12) \\
5(5) \\
73(76)\end{array}$ & .06 \\
\hline$A P O E \varepsilon 2$ genotype & 295 & $21(10)$ & $10(11)$ & .99 \\
\hline $\begin{array}{l}\text { Diagnostic class } \\
\quad 1 \\
2 \\
3 \\
4\end{array}$ & 295 & $\begin{array}{l}97(49) \\
23(12) \\
21(11) \\
58(29)\end{array}$ & $\begin{array}{c}55(57) \\
9(9) \\
9(9) \\
23(24)\end{array}$ & .59 \\
\hline Preoperative intubation & 295 & $62(31)$ & $24(25)$ & .28 \\
\hline Preoperative LOS (d) & 295 & $2.37 \pm 3.01$ & $1.62 \pm 2.19$ & .002 \\
\hline Age at first surgery (d) & 295 & $36.3 \pm 51.6$ & $57.7 \pm 59.7$ & .013 \\
\hline Hematocrit at first surgery $(\%)$ & 295 & $27.81 \pm 3.78$ & $27.67 \pm 4.53$ & .94 \\
\hline Total CPB time (min) & 295 & $64.5 \pm 39.2$ & $68.8 \pm 37.0$ & .12 \\
\hline Total DHCA time (min) & 295 & $24.4 \pm 23.0$ & $18.8 \pm 22.9$ & .04 \\
\hline Delayed sternal closure & 295 & $21(11)$ & $10(10)$ & .97 \\
\hline ECMO use & 295 & $5(3)$ & $3(3)$ & .76 \\
\hline Postoperative LOS (d) & 295 & $11.50 \pm 11.08$ & $8.85 \pm 7.22$ & .011 \\
\hline $\begin{array}{l}\text { Maternal education at } 4 \text { y } \\
\text { Less than high school } \\
\text { High school/some college } \\
\text { College } \\
\text { Graduate }\end{array}$ & 295 & $\begin{array}{l}11(6) \\
93(46) \\
65(33) \\
30(15)\end{array}$ & $\begin{array}{c}6(6) \\
27(29) \\
44(45) \\
19(20)\end{array}$ & .024 \\
\hline $\begin{array}{l}\text { Maternal SES quintile at } 4 \mathrm{y} \\
1 \\
2 \\
3 \\
4 \\
5\end{array}$ & 295 & $\begin{array}{l}6(3) \\
17(9) \\
38(19) \\
64(33) \\
71(36)\end{array}$ & $\begin{array}{c}4(4) \\
7(7) \\
15(15) \\
31(32) \\
40(42)\end{array}$ & .85 \\
\hline FSIQ score & 292 & $97.8 \pm 17.7$ & $99.3 \pm 17.9$ & - \\
\hline VMI score & 294 & $94.9 \pm 17.9$ & $93.3 \pm 16.6$ & - \\
\hline \multicolumn{5}{|l|}{ CBCL 1.5-5 measures } \\
\hline Total problems & 295 & $47.4 \pm 10.4$ & $48.8 \pm 12.9$ & - \\
\hline Internalizing problems & 295 & $48.1 \pm 10.6$ & $50.3 \pm 12.9$ & - \\
\hline Externalizing problems & 295 & $46.4 \pm 10.3$ & $46.6 \pm 11.8$ & - \\
\hline PDPs score & 295 & $54.08 \pm 6.60$ & $56.28 \pm 8.81$ & - \\
\hline
\end{tabular}

Values are presented as $\mathrm{n}(\%)$ or mean \pm standard deviation. $M B L$, Mannose-binding lectin; $\varepsilon 2$, genotype associated with poor neurodevelopmental outcomes; $A P O E \varepsilon 2$, apolipoprotein E gene genotype; $L O S$, length of stay; $C P B$, cardiopulmonary bypass; $D H C A$, deep hypothermic circulatory arrest; $E C M O$, extracorporeal membrane oxygenation; $S E S$, socioeconomic status; $F S I Q$, full scale intellectual quotient; VMI, visual motor integration; $C B C L$ 1.5-5, Child Behavior Checklist 1.5 to 5 years; $P D P s$, pervasive developmental problems. *Differences in proportions were assessed using $\chi^{2}$ tests. Tests of differences in means were performed using the Wilcoxon rank-sum test. 
groups are thus accounted for when testing the effect of genotype on each neurobehavioral outcome. Of the 6 tested neurodevelopmental outcomes, no association was noted for MBL-deficient genotype and the outcomes of FSIQ (B $=-2.78 ; P=.31)$, VMI $(\mathrm{B}=-2.89 ; P=.33)$, or CBCL 1.5-5 externalizing problems $(\mathrm{B}=1.40 ; P=.46)$ scores. Nominally significant associations were observed between the MBL-deficient genotype group and CBCL $1.5-5$ total problems $(\mathrm{B}=3.23 ; P=.097)$ and internalizing problems $(\mathrm{B}=3.33 ; P=.093)$ scores. A statistically significant association, after Bonferroni correction for 6 tests ( $\alpha=0.0083$ ), was identified between the MBL-deficient genotype group and CBCL 1.5-5 PDP score $(\mathrm{B}=3.98$; $P=.0025)$. These results are summarized in Table 2. Sensitivity analyses stratifying by self-reported race and diagnostic class did not demonstrate differences in the direction of effect for the MBL-deficient genotype.

We further sought to identify potential effect modification of the association between the MBL-deficient genotype and CBCL 1.5-5 PDP score by performing sensitivity analyses including an interaction term between MBL-deficient genotype with (separately) preoperative LOS, age at first surgery, total DHCA time, and postoperative LOS, all of which differed by MBL status (see Table 1). Of these, no interaction was noted between MBL-deficient genotype and preoperative LOS, total DHCA time, or postoperative LOS. However, an association was observed between age at first surgery and MBL-deficient genotype $\left(\gamma_{\text {interaction }}=-0.036 ; P_{\text {interaction }}=.039\right)($ Table 3$)$. Plotting of this gene $X$ environment interaction demonstrated a more deleterious neurodevelopmental effect (higher PDP scores) for neonates with the MBL-deficient genotype who underwent surgery at an early age (Figure 1). Patients who had surgery before age 30 days and were MBL deficient had a mean covariate-adjusted PDP score of 58, compared with 55 for the normal MBL group. In comparison, among patients who had surgery after age 90 days, both MBL-deficient and normal groups had a mean PDP score of 55 .

To examine whether the interaction between age at first surgery and MBL-deficient genotype reflected differences in CHD severity, we performed a sensitivity analysis for the outcome of PDP score, stratified by CHD diagnostic class $^{24}$ (Table E1). Patients with CHD class 1 or 2 did not have significant associations for either MBL-deficient genotype or age at first surgery $(P>.05)$. However, patients with CHD class 3 or 4 did have nominally significant associations between MBL deficiency and PDP scores $(P=.044$ and $P=.023$, respectively). Moreover, the interaction between MBL-deficient genotype and age at first surgery trended toward significance in both the class 3 $\left(P_{\text {interaction }}=.087\right)$ and $4\left(P_{\text {interaction }}=.057\right)$ groups. We also note that children in diagnostic class 3 and 4 tended to have an earlier age at first surgery (Figure E1). Thus,
TABLE 2. Association of autosomal dominant mannose-binding lectin (MBL) deficiency genotype with 4-year neurodevelopmental outcomes

\begin{tabular}{|c|c|c|}
\hline Outcome* & $\begin{array}{c}\beta \text { coefficient } \pm \\
\text { standard error }\end{array}$ & $P$ value \\
\hline FSIQ score $\dagger$ & $-2.78 \pm 2.75$ & .31 \\
\hline VMI score $\dagger$ & $-2.89 \pm 2.98$ & .33 \\
\hline CBCL total problems score $\ddagger$ & $3.23 \pm 1.94$ & .097 \\
\hline $\begin{array}{l}\text { CBCL persistent developmental problems } \\
\text { score } \ddagger\end{array}$ & $3.98 \pm 1.30$ & .0025 \\
\hline CBCL internalizing problems score $\ddagger$ & $3.33 \pm 1.97$ & .093 \\
\hline CBCL externalizing problems score & $1.40 \pm 1.88$ & .46 \\
\hline
\end{tabular}

FSIQ, Full Scale Intellectual Quotient; VMI, Visual Motor Integration; $C B C L$, Child Behavior Checklist 1.5 to 5 years. *Linear regression model for association of autosomal dominant MBL deficiency and 4-year neurodevelopmental outcomes adjusts for first 3 principal component eigenvectors (to adjust for genetic ancestry stratification), gestational age, birth weight, birth head circumference, APOE $\varepsilon 2$ genotype, diagnostic class (class 1 as the reference group), preoperative intubation, preoperative length of stay, hematocrit at first surgery, total minutes of deep hypothermic circulatory arrest, total minutes of cardiopulmonary bypass, delayed sternal closure, use of extracorporeal membrane oxygenation, postoperative length of stay, mother's education at 4-year follow-up (lowest education group as the reference), mother's socioeconomic status at 4-year follow-up (lowest socioeconomic quintile as the reference), and age at first surgery. $\dagger$ For FSIQ and VMI scores, scores have a mean of 100 with lower scores and negative $\beta$ coefficients corresponding to increased prevalence of problems in neurodevelopment. $¥$ For CBCL outcomes, the $T$ scores have a mean of 50 , with higher scores and $\beta$ coefficients corresponding to increased prevalence of problems in neurodevelopment.

although underpowered, we assessed a linear regression model on the outcome of PDP score using a 3-variable interaction term between CHD diagnostic class, age at first surgery, and MBL-deficient genotype $\left(P_{\text {interaction }}=.11\right)$. We also note that CHD diagnostic class did not separately modify the association of MBL-deficient genotype $\left(P_{\text {interaction }}=0.37\right)$ or age at first surgery $\left(P_{\text {interaction }}=.19\right)$ with the outcome of PDP scores.

To determine whether MBL-deficient genotype had an effect on long-term survival we performed an analysis on 422 previously described ${ }^{13,14}$ nonsyndromic CHD patients (see Table E2). From these analyses we conclude that MBL-deficient genotype is not associated with differential long-term survival (hazard ratio, $0.81 ; 95 \%$ confidence interval, $0.38-1.71 ; P=.58$ ).

\section{DISCUSSION}

We report the novel finding of a deleterious neurodevelopmental effect in CHD surgery survivors, with carriers of 1 or more copy of the $M B L 2_{\text {Gly54Asp }}$ missense variant having significantly higher scores for covariate-adjusted PDPs at 3-year follow-up $(\mathrm{B}=3.98 ; P=.0025)$. This association between MBL-deficient genotype and PDPs appeared more pronounced, with higher PDP scores in participants with both the MBL-deficient genotype and an earlier age at first surgery $\left(P_{\text {interaction }}=.039\right)$. Although MBL-deficient genotype was not significantly associated with the other tested neurodevelopmental outcomes, the trend in the direction 
TABLE 3. Full multivariable linear regression model coefficients for the outcome of pervasive developmental problems, including an interaction term between mannose-binding lectin (MBL) deficiency and age at first surgery

\begin{tabular}{|c|c|c|c|}
\hline Variable & $\beta$ coefficient \pm standard error & $\%$ variation & $P$ value \\
\hline Intercept & $55.72 \pm 12.81$ & - & - \\
\hline Male $(\%)$ & $-1.04 \pm 0.89$ & 0.23 & .24 \\
\hline Gestational age (wk) & $-0.25 \pm 0.26$ & 0.47 & .33 \\
\hline Birth weight $(\mathrm{kg})$ & $-0.0019 \pm 0.0074$ & 0.020 & .80 \\
\hline Birth head circumference $(\mathrm{cm})$ & $0.15 \pm 0.30$ & 0.060 & .63 \\
\hline $\mathrm{PC} 1$ & $-12.25 \pm 11.29$ & - & - \\
\hline $\mathrm{PC} 2$ & $12.52 \pm 10.07$ & - & - \\
\hline PC3 & $-6.26 \pm 10.84$ & - & - \\
\hline$A P O E \varepsilon 2$ genotype $(\%)$ & $3.49 \pm 1.33$ & 2.27 & .0089 \\
\hline \multicolumn{4}{|l|}{ Diagnostic class $(\%)$} \\
\hline Diagnostic class 2 & $-0.62 \pm 1.44$ & 0.20 & .67 \\
\hline Diagnostic class 3 & $1.80 \pm 1.86$ & 0.070 & .33 \\
\hline Diagnostic class 4 & $2.49 \pm 1.98$ & 0.64 & .21 \\
\hline Preoperative intubation (\%) & $0.79 \pm 1.01$ & 0.096 & .43 \\
\hline Preoperative LOS (d) & $0.11 \pm 0.17$ & 0.21 & .50 \\
\hline Hematocrit at first surgery $(\%)$ & $-0.23 \pm 0.11$ & 1.02 & .043 \\
\hline Total DHCA time (min) & $0.022 \pm 0.038$ & 0.049 & .56 \\
\hline Total CPB time (min) & $0.0038 \pm 0.018$ & 0.039 & .83 \\
\hline Delayed sternal closure $(\%)$ & $0.93 \pm 1.53$ & 0.16 & .55 \\
\hline ECMO use $(\%)$ & $2.74 \pm 3.02$ & 0.079 & .37 \\
\hline Postoperative LOS (d) & $0.12 \pm 0.055$ & 1.33 & .024 \\
\hline \multicolumn{4}{|l|}{ Maternal education at $4 \mathrm{y}$} \\
\hline High school/some college & $-3.39 \pm 2.00$ & 0.47 & .090 \\
\hline College & $-3.55 \pm 2.14$ & 0.20 & .098 \\
\hline Graduate & $-4.35 \pm 2.27$ & 0.60 & .057 \\
\hline \multicolumn{4}{|l|}{ Maternal SES quintile at $4 \mathrm{y}$} \\
\hline 2 & $2.81 \pm 2.76$ & 0.13 & .31 \\
\hline 3 & $1.89 \pm 2.56$ & 0.29 & .46 \\
\hline 4 & $0.91 \pm 2.61$ & 0.081 & .73 \\
\hline 5 & $1.87 \pm 2.67$ & 0.23 & .48 \\
\hline Age at first surgery (d) & $-0.0012 \pm 0.011$ & 0.11 & .62 \\
\hline MBL deficiency $(\%)$ & $3.98 \pm 1.30$ & 2.05 & .0079 \\
\hline MBL deficiency $\times$ age at first surgery interaction & $-0.036 \pm 0.017$ & 0.53 & .039 \\
\hline
\end{tabular}

Values are presented as mean \pm standard deviation. $P C$, Principal component (eigenvectors to adjust for differing genetic ancestry); $A P O E \varepsilon 2$, apolipoprotein $\mathrm{E}$ gene genotype associated with poor neurodevelopmental outcomes; $L O S$, length of stay; $D H C A$, deep hypothermic circulatory arrest; $C P B$, cardiopulmonary bypass; $E C M O$, extracorporeal membrane oxygenation; $S E S$, socioeconomic status; $M B L$, mannose-binding lectin.

of effect for each association was consistent with a deleterious effect on neurodevelopment for the MBL-deficient genotype group. Overall, this novel association provides additional evidence that genetic variants are important modifiers of morbidity and disability after surgery for CHD.

Other investigators have reported a deleterious effect of MBL-deficient genotype on neurological outcomes in preterm infants ${ }^{9,10}$ and that $M b l-l-$ knockout mice were at increased risk of traumatic brain injury. ${ }^{25}$ However, evidence for a protective effect of MBL loss or insufficiency has also been reported. Orsini and colleagues ${ }^{26}$ demonstrated a potential role of MBL in ischemic-reperfusion injury by illustrating that $M b l-/-$ knockout mice were protected from focal cerebral ischemia injury compared with wild type animals. ${ }^{26}$ Similar results were reported by the same group for the outcome of traumatic brain injury in a sample of 6 adult humans and 11 mice, ${ }^{27}$ and by a separate group in 135 adult stroke patients. ${ }^{28}$ Separately, in adult 


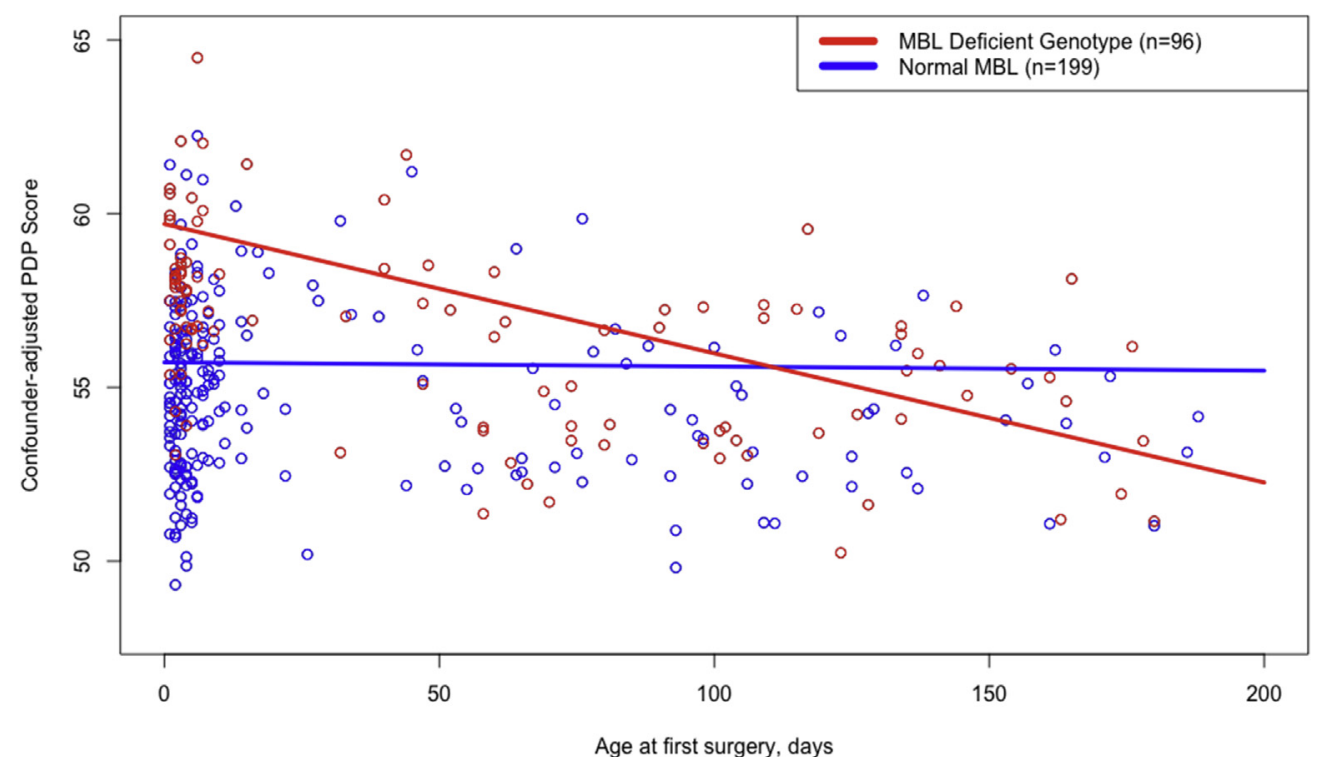

FIGURE 1. Effect of mannose-binding lectin $(M B L)$-deficient genotype is modified by age at first surgery, with infants receiving surgery at an early age having significantly higher pervasive developmental problems $(P D P)$ scores $\left(P_{\text {interaction }}=.039\right)$. Predicted values of Child Behavior Checklist 1.5 to 5 years PDP score are 59.7 to $0.0372 \times$ (age at first surgery, in days) for the MBL-deficient genotype group and $55.72-0.0012 \times($ age at first surgery, in days) for the normal MBL group.

cardiovascular disease, low MBL levels have been reported to be protective against multiorgan dysfunction ${ }^{29}$ and postoperative myocardial infarction ${ }^{30}$ after cardiac surgery.

Compared with adult and animal studies, investigations into the effect of MLB-deficient genotype in pediatric populations have more consistently reported deleterious effects. Notably, low MBL levels and MBL-deficient genotypes were noted in a higher proportion of premature neonates compared with term children. ${ }^{31}$ In addition, among premature neonates, those with low MBL levels at baseline had significantly increased risk of adverse neurologic outcomes. ${ }^{32}$ Further, MBL-deficient genotype has been associated with increased risk of respiratory distress syndrome, ${ }^{33}$ sepsis, ${ }^{9}$ and adverse neurological outcomes in neonates. ${ }^{10}$ Within this context of pediatric disease, our results of a deleterious neurodevelopmental effect of MBL-deficient genotype are consistent with prior literature. We speculate that the potential mechanism through which MBLdeficient genotype exerts its adverse neurodevelopmental effects is indirect: through increased infection risk. This mechanism is strengthened through evidence of decreased complement (ie, C3/C4) levels in MBL-deficient genotype patients, ${ }^{32}$ and the consistent literature in pediatric populations that suggest an association between low MBL levels and increased rates of childhood infection, ${ }^{6,7}$ sepsis, ${ }^{9}$ and respiratory distress syndrome. ${ }^{33}$ However, we also report from our data a decrease in both preoperative and postoperative LOS for the MBL-deficient genotype group, suggesting that, similar to the adult cardiovascular surgery literature, ${ }^{29,30}$ decreased $\mathrm{MBL}$ is protective against immediate injury after cardiac surgery. Further research is necessary to clarify and further elucidate the role of MBL in the immediate setting after cardiac surgery, and in the longer-term with regard to neurologic and neurodevelopmental outcomes.

Some limitations of this study should be considered. First, statistical power was limited due to the size of the study and lack of comparable cohorts. We addressed this by limiting the number of primary tests to 6 neurodevelopmental outcomes that broadly assessed function and behavior. However, it is possible that other significant associations in untested neurodevelopmental outcomes were overlooked through this approach. Second, we were unable to measure MBL protein levels directly due to a lack of serum in this long-standing cohort. However, because the studied genetic variant (rs1800450 causing the $M B L 2_{\text {Gly54Asp }}$ missense variant and resulting autosomal dominant MBL deficiency) is expected to reduce MBL levels by $90 \%,{ }^{6,7}$ we believe it is a suitable proxy for MBL levels in vivo. Finally, because these analyses were of 4-year neurodevelopmental outcomes requiring followup, there is the possibility of survivor bias. To identify if our results reflected survival bias and potentially worse neurodevelopmental outcomes in survivors with MBL deficiency, we performed a secondary analysis and determined that MBL-deficient genotype was not associated with differential survival.

In addition to the above limitations of our work, we note that our identified effect modification between MBLdeficient genotype and age at first surgery on the outcome 
of PDP score could also be affected by the observation that those who undergo surgery before age 30 days tend to have more severe $\mathrm{CHD}^{34}$ (Figure E1). Because patients with more severe CHD also tend to have poorer neurodevelopmental outcomes, ${ }^{12}$ hypothetical survival of these children could have potentially demonstrated a more deleterious interaction between age at first surgery and MBLdeficient genotype on neurodevelopment. To assess this possibility, we performed a sensitivity analysis that demonstrated stronger associations for both MBL-deficient genotype and the interaction between age at first surgery and MBL deficiency with PDP scores in the class 3 and 4 children, who have 1 ventricle with (class 4 ) or without (class 3 ) aortic arch obstruction. While this sensitivity analysis was suggestive of further effect modification by CHD severity, modeling of 2-way (CHD class $\times$ age at first surgery or CHD class $\times$ MBL deficiency) and 3-way (CHD class $\times$ age at first surgery $\times$ MBL-deficient genotype) were not suggestive of significance $\left(P_{\text {interaction }}>.10\right)$. Thus, although we conclude that CHD severity did not affect the deleterious association of MBL deficiency with PDP scores, CHD severity remains an important topic for future follow-up work in the study of neurodevelopment after surgical palliation.

\section{CONCLUSIONS}

We report the novel association of MBL-deficient genotype and poor neurodevelopmental outcomes in the setting of CHD. We further identified effect modification by age at first surgery, whereby children who underwent surgery at an early age and had the MBL-deficient genotype were at greater risk of deleterious neurodevelopmental outcomes. Given the importance of neurodevelopmental function in CHD survivors, validation of these results could lead to novel preventative and risk assessment strategies to decrease the long-term morbidity of CHD surgery.

\section{Conflict of Interest Statement}

Ms McDonald-McGinn has presented lectures on 22q11.2 deletion syndrome for Natera. All other authors have nothing to disclose with regard to commercial support.

The authors thank the children and families for their participation. Genotyping was performed by the Center for Applied Genomics at the Children's Hospital of Philadelphia.

\section{References}

1. Bellinger DC, Jonas RA, Rappaport LA, Wypij D, Wernovsky G, Kuban KC, et al. Developmental and neurologic status of children after heart surgery with hypothermic circulatory arrest or low-flow cardiopulmonary bypass. $N$ Engl J Med. 1995;332:549-55.

2. Bellinger DC, Wypij D, duPlessis AJ, Rappaport LA, Jonas RA, Wernovsky G, et al. Neurodevelopmental status at eight years in children with dextrotransposition of the great arteries: The Boston Circulatory Arrest Trial. J Thorac Cardiovasc Surg. 2003;126:1385-96.
3. Gaynor JW, Nord AS, Wernovsky G, Bernbaum J, Solot CB, Burnham N, et al. Apolipoprotein E genotype modifies the risk of behavior problems after infant cardiac surgery. Pediatrics. 2009;124:241-50.

4. Ikeda K, Sannoh T, Kawasaki N, Kawasaki T, Yamashina I. Serum lectin with known structure activates complement through the classical pathway. J Biol Chem. 1987;262:7451-4

5. Mogues T, Ota T, Tauber AI, Sastry KN. Characterization of two mannosebinding protein cDNAs from rhesus monkey (Macaca mulatta): structure and evolutionary implications. Glycobiology. 1996;6:543-50.

6. Super M, Thiel S, Lu J, Levinsky RJ, Turner MW. Association of low levels of mannan-binding protein with a common defect of opsonisation. Lancet. 1989; 2:1236-9.

7. Sumiya M, Super M, Tabona P, Levinsky RJ, Arai T, Turner MW, et al. Molecular basis of opsonic defect in immunodeficient children. Lancet. 1991;337:1569-70.

8. Shi L, Takahashi K, Dundee J, Shahroor-Karni S, Thiel S, Jensenius JC, et al. Mannose-binding lectin-deficient mice are susceptible to infection with Staphylococcus aureus. J Exp Med. 2004;199:1379-90.

9. Koroglu OA, Onay H, Erdemir G, Yalaz M, Cakmak B, Akisu M, et al. Mannosebinding lectin gene polymorphism and early neonatal outcome in preterm infants. Neonatology. 2010;98:305-12.

10. Auriti C, Prencipe G, Caravale B, Coletti MF, Ronchetti MP, Piersigilli F, et al. MBL2 gene polymorphisms increase the risk of adverse neurological outcome in preterm infants: a preliminary prospective study. Pediatr Res. 2014;76:464-9.

11. Gaynor JW, Gerdes M, Zackai EH, Bernbaum J, Wernovsky G, Clancy RR, et al. Apolipoprotein E genotype and neurodevelopmental sequelae of infant cardiac surgery. J Thorac Cardiovasc Surg. 2003;126:1736-45.

12. Gaynor JW, Wernovsky G, Jarvik GP, Bernbaum J, Gerdes M, Zackai E, et al. Patient characteristics are important determinants of neurodevelopmental outcome at one year of age after neonatal and infant cardiac surgery. J Thorac Cardiovasc Surg. 2007;133:1344-53.e3.

13. Kim DS, Kim JH, Burt AA, Crosslin DR, Burnham N, McDonald-McGinn DM, et al. Patient genotypes impact survival after surgery for isolated congenital heart disease. Ann Thorac Surg. 2014;98:104-10.

14. Kim DS, Kim JH, Burt AA, Crosslin DR, Burnham N, Kim CE, et al. Burden of potentially pathologic copy number variants is higher in children with isolated congenital heart disease and significantly impairs covariate-adjusted transplant-free survival. J Thorac Cardiovasc Surg. 2016;151:1147-51.e4.

15. Kim DS, Stanaway IB, Rajagopalan R, Bernbaum JC, Solot CB, Burnham N, et al. Results of genome-wide analyses on neurodevelopmental phenotypes at four-year follow-up following cardiac surgery in infancy. PLoS One. 2012;7:e45936.

16. Wechsler D. Wechsler Preschool and Primary Scale of Intelligence-Third Edition (WPPSI - III). San Antonio, TX: Pearson Clinical; 2002.

17. Beery KE, Buktenica NA. In: Beery-Buktenica Developmental Test of VisualMotor Integration, Sixth Edition, the (BEERY VMI). San Antonio, TX: Pearson Clinical; 2010.

18. Achenbach TM, Rescorla LA. ASEBA Child Behavior Checklist for Ages 1.5-5 (CBCL/1.5-5) and ASEBA Caregiver-Teacher Report Form for Ages 1.5-5 (CTRF). Lutz, FL: Psychological Assessment Resources; 2001.

19. Mazefsky CA, Anderson R, Conner CM, Minshew N. Child Behavior Checklist scores for school-aged children with autism: preliminary evidence of patterns suggesting the need for referral. J Psychopathol Behav Assess. 2010;33:31-7.

20. Purcell S, Neale B, Todd-Brown K, Thomas L, Ferreira MA, Bender D, et al. PLINK: a tool set for whole-genome association and population-based linkage analyses. Am J Hum Genet. 2007;81:559-75.

21. Gaynor JW, Kim DS, Arrington CB, Atz AM, Bellinger DC, Burt AA, et al. Validation of association of the apolipoprotein E $\varepsilon 2$ allele with neurodevelopmental dysfunction after cardiac surgery in neonates and infants. $J$ Thorac Cardiovasc Surg. 2014;148:2560-6.

22. Kosoy R, Nassir R, Tian C, White PA, Butler LM, Silva G, et al. Ancestry informative marker sets for determining continental origin and admixture proportions in common populations in America. Hum Mutat. 2009;30:69-78.

23. Price AL, Patterson NJ, Plenge RM, Weinblatt ME, Shadick NA, Reich D. Principal components analysis corrects for stratification in genome-wide association studies. Nat Genet. 2006;38:904-9.

24. Clancy RR, McGaurn SA, Wernovsky G, Spray TL, Norwood WI, Jacobs ML, et al. Preoperative risk-of-death prediction model in heart surgery with deep hypothermic circulatory arrest in the neonate. J Thorac Cardiovasc Surg. 2000;119: 347-57.

25. Yager PH, You Z, Qin T, Kim HH, Takahashi K, Ezekowitz AB, et al. Mannose binding lectin gene deficiency increases susceptibility to traumatic brain injury in mice. J Cereb Blood Flow Metab. 2008;28:1030-9. 
26. Orsini F, Villa P, Parrella S, Zangari R, Zanier ER. Targeting mannose binding lectin confers long lasting protection with a surprisingly wide therapeutic window in cerebral ischemia. Circulation. 2012;126:1484-94.

27. Longhi L, Orsini F, De Blasio D, Fumagalli S, Ortolano F, Locatelli M, et al. Mannose-binding lectin is expressed after clinical and experimental traumatic brain injury and its deletion is protective. Crit Care Med. 2014; 42:1910-8.

28. Cervera A, Planas AM, Justicia C, Urra Z, Jensenius JC, Torres F, et al. Genetically-defined deficiency of mannose-binding lectin is associated with protection after experimental stroke in mice and outcome in human stroke. PLoS One. 2010; 5:e8433.

29. Bilgin YM, Brand A, Berger SP, Daha MR, Roos A. Mannose-binding lectin is involved in multiple organ dysfunction syndrome after cardiac surgery: effects of blood transfusions. Transfusion. 2008;48:601-8.

30. Collard CD, Shernan SK, Fox AA, Bernig T, Chanock SJ, Vaughn WK, et al. The MBL2 "LYQA secretor" haplotype is an independent predictor of postoperative myocardial infarction in whites undergoing coronary artery bypass graft surgery. Circulation. 2007;116(11 Suppl):I106-12.
31. Frakking FNJ, Brouwer N, Zweers D, Merkus MP, Kuijpers MP, Offringa M et al. High prevalence of mannose-binding lectin (MBL) deficiency in premature neonates. Clin Exp Immunol. 2006;145:5-12.

32. Xue J, Liu A-H, Zhao B, Si M, Li Y-Q. Low levels of mannose-binding lectin at admission increase the risk of adverse neurological outcome in preterm infants: a 1-year follow-up study. J Matern Fetal Neonatal Med. 2015;29:1425-9.

33. Speletas M, Gounaris A, Sevdali E, Kompoti M, Konstandtinidi K, Sokou R, et al. MBL2 Genotypes and their associations with MBL levels and NICU morbidity in a cohort of Greek neonates. J Immunol Res. 2015 2016:478412.

34. Gaynor JW, Mahle WT, Cohen MI, Ittenbach RF, De Campli WM, Steven JM, et al. Risk factors for mortality after the Norwood procedure. Eur J Cardiothorac Surg. 2002;22:82-9.

Key Words: congenital heart disease, neurodevelopment, genetics, MBL2, mannose-binding lectin, gene-byenvironment interaction, gene-environment interactions 


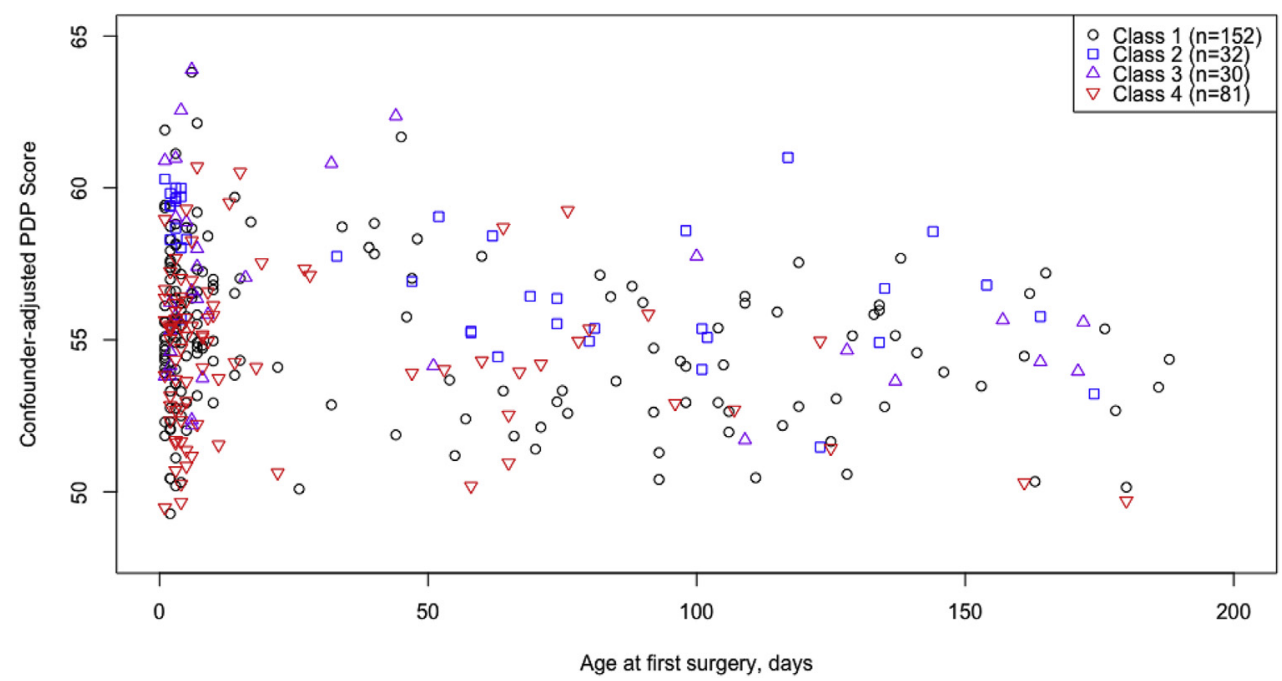

FIGURE E1. Distribution of age at first surgery and covariate-adjusted pervasive developmental problem (PDP) scores by congenital heart disease (CHD) diagnostic class.

TABLE E1. Sensitivity analysis by congenital heart disease diagnostic class on the association between age at first surgery and mannose-binding lectin (MBL) deficiency on the outcome of pervasive developmental problems

\begin{tabular}{|c|c|c|}
\hline Variable & $\beta$ coefficient \pm standard error & $P$ value $*, \dagger$ \\
\hline \multicolumn{3}{|l|}{ Diagnostic class $1(n=152)$} \\
\hline Age at first surgery (d) & $-0.00083 \pm 0.014$ & .95 \\
\hline MBL deficiency $(\%)$ & $2.66 \pm 2.15$ & .76 \\
\hline MBL deficiency $\times$ age at first surgery interaction & $0.0098 \pm 0.022$ & .66 \\
\hline \multicolumn{3}{|l|}{ Diagnostic class $2(n=32)$} \\
\hline Age at first surgery (d) & $-0.0042 \pm 0.091$ & .65 \\
\hline MBL deficiency $(\%)$ & $4.47 \pm 4.90$ & .46 \\
\hline MBL deficiency $\times$ age at first surgery interaction & $-0.028 \pm 0.094$ & .77 \\
\hline \multicolumn{3}{|l|}{ Diagnostic class $3(n=30)$} \\
\hline Age at first surgery $(\mathrm{d})$ & $-0.018 \pm 0.089$ & .085 \\
\hline MBL deficiency $(\%)$ & $6.50 \pm 2.77$ & .044 \\
\hline MBL deficiency $\times$ age at first surgery interaction & $-0.025 \pm 0.15$ & .087 \\
\hline \multicolumn{3}{|l|}{ Diagnostic class $4(n=81)$} \\
\hline Age at first surgery $(\mathrm{d})$ & $-0.036 \pm 0.18$ & .045 \\
\hline MBL deficiency $(\%)$ & $6.18 \pm 2.66$ & .023 \\
\hline MBL deficiency $\times$ Age at first surgery interaction & $-0.039 \pm 0.20$ & .057 \\
\hline
\end{tabular}


TABLE E2. Multivariable Cox proportional hazards model coefficients for the association between mannose-binding lectin (MBL) genotype and long-term survival ( $\mathrm{N}=422 ; 47$ total deaths during follow-up)

\begin{tabular}{|c|c|c|}
\hline Variable & Hazard ratio $(95 \%$ confidence interval) & $P$ value \\
\hline Male $(\%)$ & $1.18(0.61-2.27)$ & .62 \\
\hline Gestational age (wk) & $1.07(0.87-1.32)$ & .51 \\
\hline Birth weight $(\mathrm{kg})$ & $0.56(0.28-1.13)$ & .11 \\
\hline PC1 & - & - \\
\hline $\mathrm{PC} 2$ & - & - \\
\hline PC3 & - & - \\
\hline Age at first surgery (d) & $0.99(0.99-1.01)$ & .90 \\
\hline$A P O E \varepsilon 2$ genotype $(\%)$ & $1.55(0.72-3.37)$ & .27 \\
\hline \multicolumn{3}{|l|}{ Diagnostic class $(\%)$} \\
\hline Diagnostic class 2 & $0.50(0.056-4.51)$ & .54 \\
\hline Diagnostic class 3 & $5.79(1.66-20.18)$ & .0058 \\
\hline Diagnostic class 4 & $6.57(2.31-18.65)$ & .00041 \\
\hline Preoperative intubation (\%) & $0.65(0.32-1.31)$ & .23 \\
\hline Preoperative LOS (d) & $0.94(0.79-1.13)$ & .51 \\
\hline Hematocrit at first surgery $(\%)$ & $1.02(0.94-1.09)$ & .69 \\
\hline Total DHCA time (min) & $1.02(1.01-1.04)$ & .0037 \\
\hline Total CPB time (min) & $1.00(0.99-1.01)$ & .73 \\
\hline Delayed sternal closure $(\%)$ & $1.02(0.45-2.30)$ & .97 \\
\hline ECMO use $(\%)$ & $6.39(2.76-14.80)$ & .000015 \\
\hline MBL deficiency $(\%)$ & $0.81(0.38-1.71)$ & .58 \\
\hline
\end{tabular}

$P C$, Principal component (eigenvectors to adjust for differing genetic ancestry); $A P O E \varepsilon 2$, apolipoprotein $\mathrm{E}$ gene genotype; $L O S$, length of stay; $D H C A$, deep hypothermic circulatory arrest; $C P B$, cardiopulmonary bypass; $E C M O$, extracorporeal membrane oxygenation; $M B L$, mannose-binding lectin. 